\title{
STRUCTURED MORPHOSPACES AND DISCONTINUOUS EVOLUTION
}

REIF*, Wolf-Ernst, Institut für Geologie und Paläontologie, Universität Tübingen, Sigwartstraße 10, 72076 Tübingen, Federal Republic of Germany; WEISHAMPEL, David B., Department of Cell Biology and Anatomy, The Johns Hopkins University School of Medicine, Baltimore, Maryland 21205, U.S.A.

The concept of an $\mathrm{n}$-dimensional morphospace was developed for two basic reasons. The first objective is to demonstrate and plot all biological forms that can be produced by a growth program which has $\mathrm{n}$ different parameters; the whole spectrum of possible forms is produced by varying all of these parameters. Second, the morphospace concept provides the basis for the identification of those areas of a theoretical morphospace that have been invaded by evolution as a whole or by individual higher taxa; this can be used as a comparative measure of the morphological diversity of higher taxa.

Here we demonstrate the application of the morphospace concept in the context of continuous versus discontinuous evolution. Concepts of unconstrained evolution necessarily assume that the morphospace of all possible organic forms is unstructured and that evolutionary change is possible in any direction of the morphospace. Other concepts of evolution regard the morphospace as structured with discrete inhabitable and non-inhabitable areas. It can be shown that the common theme of typological, saltational, macroevolutionist, and structuralist theories of evolution is the attempt to demonstrate why the morphospace of all possible organic forms is structured in a law-like fashion. 